ARTICLE

\title{
Connexin-46/50 in a dynamic lipid environment resolved by CryoEM at $1.9 \AA$
}

Jonathan A. Flores ${ }^{1,5}$, Bassam G. Haddad ${ }^{2,5}$, Kimberly A. Dolan ${ }^{2,4,5}$, Janette B. Myers ${ }^{2}$, Craig C. Yoshioka ${ }^{3}$, Jeremy Copperman (10 ${ }^{3}$, Daniel M. Zuckerman (i) ${ }^{3} \&$ Steve L. Reichow (1) ${ }^{1,2 凶}$

Gap junctions establish direct pathways for cells to transfer metabolic and electrical messages. The local lipid environment is known to affect the structure, stability and intercellular channel activity of gap junctions; however, the molecular basis for these effects remains unknown. Here, we incorporate native connexin-46/50 (Cx46/50) intercellular channels into a dual lipid nanodisc system, mimicking a native cell-to-cell junction. Structural characterization by CryoEM reveals a lipid-induced stabilization to the channel, resulting in a $3 \mathrm{D}$ reconstruction at $1.9 \AA$ resolution. Together with all-atom molecular dynamics simulations, it is shown that $C \times 46 / 50$ in turn imparts long-range stabilization to the dynamic local lipid environment that is specific to the extracellular lipid leaflet. In addition, 400 water molecules are resolved in the CryoEM map, localized throughout the intercellular permeation pathway and contributing to the channel architecture. These results illustrate how the aqueous-lipid environment is integrated with the architectural stability, structure and function of gap junction communication channels.

\footnotetext{
${ }^{1}$ Department of Chemical Physiology and Biochemistry, Oregon Health and Science University, Portland, OR 97239, USA. ${ }^{2}$ Department of Chemistry, Portland State University, Portland, OR 97201, USA. ${ }^{3}$ Department of Biomedical Engineering, Oregon Health and Science University, Portland, OR 97239, USA. ${ }^{4}$ Present address: Biophysics Graduate Group, University of California, Berkeley, CA 94720, USA. ${ }^{5}$ These authors contributed equally: Jonathan A. Flores, Bassam G. Haddad, Kimberly A. Dolan. ${ }^{凶}$ email: reichow@pdx.edu
} 
T he connexins are a family of transmembrane proteins (21 isoforms in human) that form intercellular channels for cell-to-cell communication ${ }^{1,2}$. These intercellular channels establish a $\sim 1.4 \mathrm{~nm}$ pore that couples the cytoplasms of neighboring cells and enable direct passage of electrical and smallmolecule signals (such as ions, second messengers, hormones, and metabolites) ${ }^{3}$ and therapeutic agents ${ }^{4}$. Tens to 1000 s of connexin channels may assemble together to form large hexagonally packed arrays, a.k.a., plaques, known as gap junctions. In this way, gap junctions enable the near instantaneous response of electrical synapses in the brain and heart, and contribute to the long-range signaling and metabolic coupling of most tissues. Because of these fundamental roles, aberrant gap junctional coupling is associated with a variety of human diseases, including blindness, deafness, skin disorders, arrhythmia, stroke, and cancers $^{5-7}$.

Gap junction intercellular communication is facilitated by a unique macromolecular architecture, where intercellular channels directly couple the cytoplasms of two neighboring cells. The lipid bilayers of opposing cells are separated by a characteristic gap of $\sim 3.5 \mathrm{~nm}^{8}$, a feature for which these structures were first recognized in electron micrographs of cell sections $s^{9,10}$. Furthermore, large-scale gap junctional plaque formation is dependent upon a dense mosaic of protein-lipid interactions. In vitro reconstitution studies have established that plaque assembly and intercellular channel function are dependent on the lipid environment ${ }^{10-15}$. However, the molecular basis for these effects remain largely unknown, due to the lack of high-resolution structural information within a lipid bilayer.

Here we present an electron cryo-microscopy (CryoEM) structure of native connexin-46/50 (Cx46/50) intercellular channels stabilized in a dual lipid nanodisc system at $1.9 \AA$ resolution -providing a near-atomic level of detail for this class of membrane channels. These structural results are coupled with all-atom molecular dynamics (MD) simulation studies, which together reveal many architectural and proposed functional features of the connexin channels. Cx46/50 is shown to have a remarkable influence on the local lipid environment, effectively inducing a phase separation (to the gel state) that is specific to the extracellular lipid leaflet of the two opposed membranes. Threedimensional (3D) heterogeneity analysis of the CryoEM data identified multiple lipid configurations that co-exist within the dynamic lattice of stabilized lipids, which is further detailed by MD. In addition, 400 water molecules are resolved in the CryoEM map, localized at architectural and functionally important sites. Together, this work uncovers previously unrecognized roles of the aqueous-lipid environment in stabilizing the structure and assembly of the gap junctions, and suggest $\mathrm{Cx} 46 / 50$ plays an important role in shaping the properties of local membrane environment.

\section{Results}

Structural overview of $\mathrm{Cx} 46 / 50$ in a lipid environment. Native (heteromeric/heterotypic) $\mathrm{Cx} 46 / 50$ intercellular channels were purified from mammalian lens tissue (obtained from sheep) ${ }^{16}$. Freshly purified channels were reconstituted into self-assembling lipid nanodiscs containing pure dimyristoyl phosphatidylcholine (DMPC) at room temperature $\left(\sim 25^{\circ} \mathrm{C}\right)$, supported by the membrane scaffold protein 1E1 (MSP1E1) ${ }^{17}$ (see "Methods"). Under optimized conditions, the reconstitution resulted in a monodispersed population of intercellular channels embedded into a pair of lipid nanodiscs, as assessed by size-exclusion chromatography (SEC) and negative-stain electron microscopy (EM) (Supplementary Fig. 1).
Structure determination by high-resolution single-particle CryoEM resulted in a high-quality $3 \mathrm{D}$ reconstruction, with an overall resolution of $1.90 \AA$ (gold-standard Fourier-shell correlation (FSC)) (Fig. 1a, b, Supplementary Figs. 2 and 3, and Supplementary Movie 1). The quality of the CryoEM map allowed for a detailed stereochemical structural refinement of both $\mathrm{Cx} 46$ and Cx50 (Fig. 1b, Supplementary Table 1, and Supplementary Fig. 3). The heteromeric pattern(s) of Cx46/50 coassembly remain unresolved, following various attempts at computational image classification (see "Methods"). Nevertheless, atomic models of both $\mathrm{Cx} 50$ and $\mathrm{Cx} 46$ isoforms were equally well-fit into the D6-symmetrized CryoEM map, reflecting their close sequence and structural similarities, $89 \%$ sequence similarity over the structured regions, and a resulting $0.17 \AA$ backbone rootmean-squared deviation (r.m.s.d.) (see "Methods" and Supplementary Fig. 3 for details and limitations regarding the heterogeneity of the natively isolated specimen).

$\mathrm{Cx} 46 / 50$ is captured in the stabilized open state, as previously described $^{16}$ (backbone Ca r.m.s.d. $=0.49-0.56 \AA$ and Supplementary Fig. 5), and exposes many undescribed features of the connexin channels that are detailed below. Intercellular channels are constructed by a dodecameric (12-mer) assembly, with six subunits assembled into "hemi-channels" that dock together through extracellular domains, resulting in a continuous $\sim 1.4 \mathrm{~nm}$ pore for intercellular permeation (Fig. 1a, c and Supplementary Movie 2). The distance separating the two lipid nanodisc densities is $\sim 3.5 \mathrm{~nm}$ (Fig. 1a, c), matching that observed by X-ray diffraction on native gap junctional plaques ${ }^{8}$.

Each monomer consists of four transmembrane helices (TM1-4), two extracellular loops (EC1-2) that form the sites of docking interaction, and an amphipathic $\mathrm{N}$-terminal helix (NTH), implicated in channel selectivity/gating. The NTH is well resolved in the stabilized open state, as we have previously described $^{16}$ (Fig. 1c, d and Supplementary Movie 3). However, the significant enhancement in resolution allowed for a detailed refinement of side chain conformations and notable improvement in precision at functional sites, including the NTH domain and the EC1/2 docking sites (Supplementary Figs. 3 and 5). Furthermore, the quality of the CryoEM map allowed for modeling previously unresolved regions of TM2 and TM3, which effectively extend the cytoplasmic vestibule of the channel by $\sim 20 \AA$, as compared to our previous model (Fig. 1c, d), significantly augmenting the electrostatic environment of the pore entrance (Supplementary Fig. 5). The intracellular loop (ICL) and C-terminal domain (CTD) remain unresolved, presumably due to intrinsic disorder of these regulatory domains ${ }^{16,18,19}$.

Perhaps the most remarkable features of the CryoEM map, however, are the non-protein components of the cell-to-cell junction that are now resolved. A bouquet of 15 ordered lipid acyl chains is held in place by each of the 12 connexin subunits, which appear to buttress the channel assembly by filling a cavity formed at the lateral subunit interfaces (Fig. la, c, d; blue). Surprisingly, acyl-chain densities are observed well beyond the first layer of annular lipids that directly interact with the TM domains (primarily TM4 and TM3 of a neighboring subunit) (Fig. 1c, d and Supplementary Fig. 6), suggesting Cx46/50 has a long-range effect on the stability and biophysical properties of the membrane. Remarkably, all of the resolved lipid densities in the CryoEM map are specifically localized to the extracellular leaflet of the bilayer, indicating a selective interaction with the local lipid environment. In contrast, individual lipids are not resolved in the intracellular leaflet, even at lower contour levels (Fig. 1a and Supplementary Fig. 2) presumably due to intrinsic disorder and/ or lack of specific interaction with this region of the channel. 

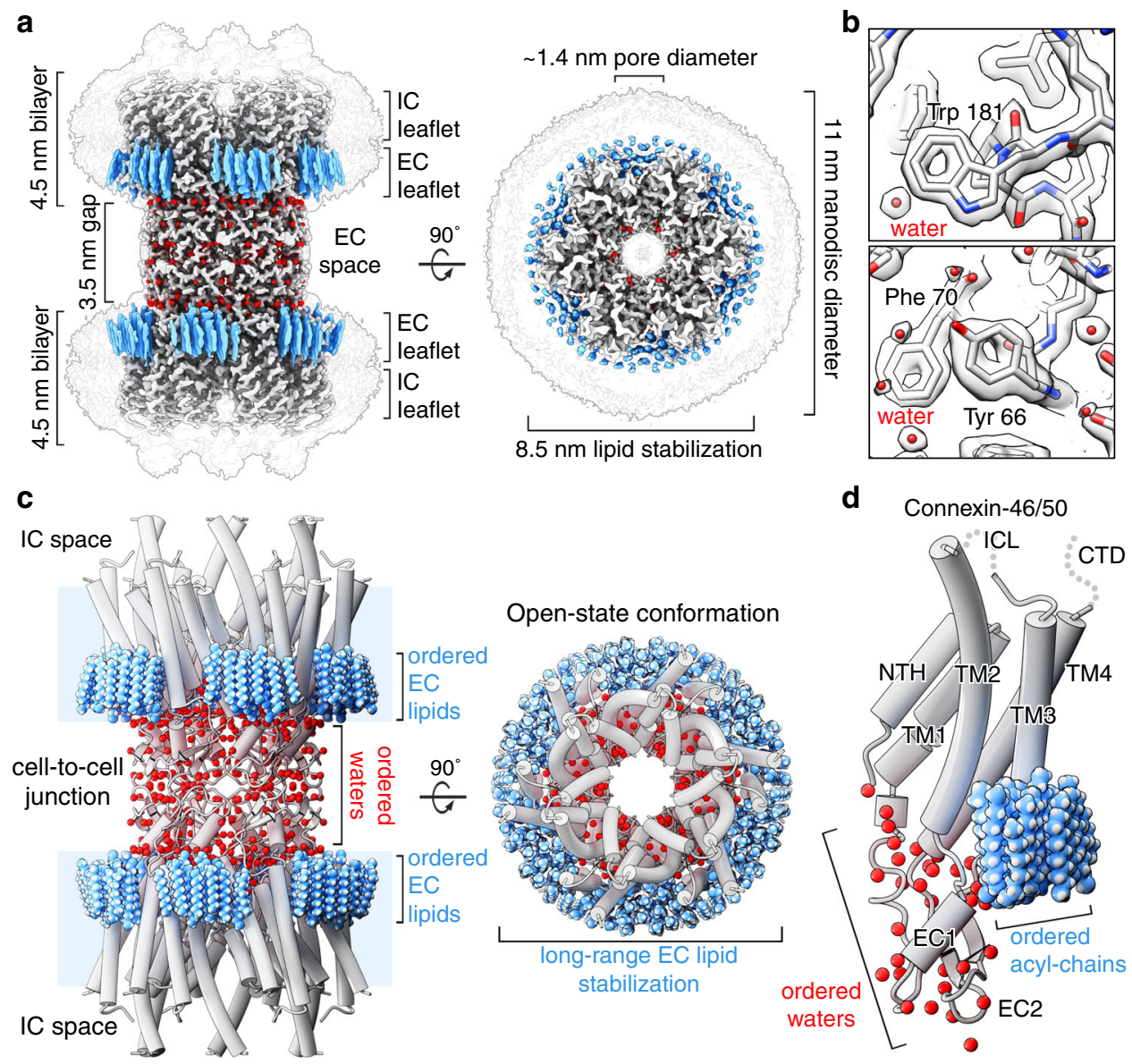

Fig. 1 Structure of connexin-46/50 in lipid nanodiscs by CryoEM. a CryoEM 3D reconstruction of Cx46/50 (white) in an open-state conformation, with resolved lipid acyl chains (blue) and water molecules (red). Transparent silhouette displays the map at low contour to illustrate the dimensions of the lipid nanodisc densities, with intracellular (IC) and extracellular (EC) lipid leaflets indicated. b Zoom views of the CryoEM map and fitted atomic models, showing high-resolution features observed at $1.9 \AA$ resolution. c Model of Cx46/50 (cylinder representation) with extracellular (EC) lipids and ordered water molecules displayed (spheres). d Cx46/50 monomer, 15 bound lipids, and 33 waters associated with each subunit. Domains labeled for transmembrane helices (TM1-4), extracellular loops (EC1-2), and N-terminal helix (NTH). The intracellular loop (ICL) and C-terminal domain (CTD) are not resolved, indicated by dotted lines.

In addition to stabilized lipids, 396 ordered water molecules are resolved throughout the channel (33 waters per subunit) (Figs. 1 and 2 red, and Supplementary Fig. 6). Waters are found at both solvent accessible and buried sites within the core of the channel, apparently contributing to the permeation pathway and structural integrity of the channel assembly (Figs. 1 and 2). The assignment of water densities was validated by assessment of hydrogenbonding patterns ( $<4 \AA$ A donor-acceptor distances) and supported by comparison to all-atom equilibrium MD simulations conducted in the presence of explicit water and $150 \mathrm{mM} \mathrm{NaCl}$ or $\mathrm{KCl}$ (see "Methods" and Supplementary Figs. 7 and 8). There was no clear evidence that the resolved solvent sites correlated with lowaffinity-ion-binding sites observed by $\mathrm{MD}^{16}$. In the following sections, we describe these resolved features in further detail and discuss their potential structural and functional roles.

Roles of stabilized waters in $\mathrm{Cx} 46 / 50$ intercellular channels. Gap junctions establish aqueous pathways that allow a variety of cytosolic substrates, less than $\sim 1 \mathrm{kDa}$ in size, to permeate from cell to cell ${ }^{20}$. The permeation pathway is established by the porelining NTH domain, TM1/2, and EC1 domains (Fig. 1c, d). Within the channel pore of $\mathrm{Cx} 46 / 50$, there are 108 waters bound at solvent-exposed sites (9 per subunit). Pore-bound waters localize to regions of the EC1 domain and TM1 parahelix, and mediate an extensive network of h-bonding interactions, involving D42, F43 ( $\pi$-bonding), E48, Q49, N63, and R76 in Cx50 (positions 42 and 43 are Glu in $\mathrm{Cx} 46$ ), and several protein backbone interactions (Fig. 2a, b and Supplementary Fig. 6).

EC1 and the TM1 parahelix contribute to the selectivity, conductance, and slow (loop) voltage-gating mechanisms of Cx46/50 $16,21-23$ and other connexins ${ }^{24-29}$, and are implicated in $\mathrm{Ca}^{2+}$ regulation in $\mathrm{Cx} 26$ by X-ray crystallography ${ }^{19}$, MD studies ${ }^{27,30}$, and by functional mutation studies of $\mathrm{Cx} 46^{30}$. As such, these pore-lining waters may functionally contribute to these mechanisms, e.g., by orienting or extending the hydrogenbonding potential of amino-acid sidechains involved in the coordination of substrates (or regulatory ions), buffering the electrostatic properties of the channel pore, or integrating the electrostatic network that is proposed to couple EC1/TM1 to the fast (NTH) voltage-gating domain ${ }^{31,32}$.

On the extracellular surface of the channel, symmetry-related rings of tightly bound water molecules are organized at the extracellular aqueous-lipid boundary (Figs. 1a, $c$ and 2a, c). In the ensemble CryoEM map, the phosphatidylcholine (PC) lipid head groups are not resolved (due to local disorder described in the following sections). Nevertheless, these stabilized rings of water are nominally positioned at the acyl-head group boundary of the extracellular lipid leaflet. These waters are stabilized by hydrogen 

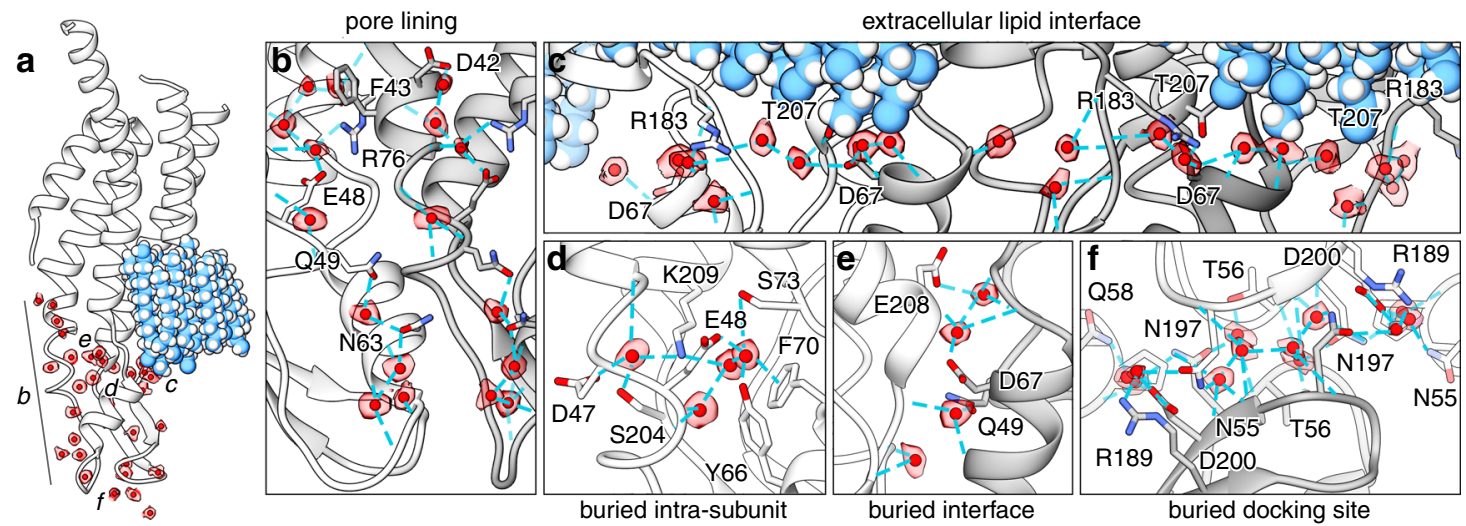

Fig. 2 Ordered water molecules resolved in Cx46/50 by CryoEM. a Cx46/50 subunit with segmented CryoEM density of waters overlaid in transparency (colored as in Fig. 1). Labels in a indicate position of the various zoom views, presented in $\mathbf{b}-\mathbf{f}$, showing water molecules bound to $\mathbf{b}$ pore-lining sites, c extracellular lipid interface, $\mathbf{d}$ buried intra-subunit sites, e buried subunit interface sites, and $\mathbf{f}$ buried cell-to-cell docking sites. In $\mathbf{b}-\mathbf{f}$, amino-acid sidechains forming hydrogen bonds to water are displayed (blue dashed lines) and labeled using Cx50 numbering.

bonds with EC1/2 residues (D67, R183/Q171, and T207/T195 in $\mathrm{Cx} 50 / \mathrm{Cx} 46)$ and expected to be further coordinated through nonspecific interactions with the phospho-glycerol backbone of the extracellular PC lipids (Fig. 2c; and discussed below).

The EC1/2 domains appear to be the most well-ordered region of the channel, as reflected by local resolution of the CryoEM density map (Supplementary Fig. 3) and root-mean-square fluctuation (r.m.s.f.) analysis of MD trajectories (Supplementary Fig. 7) This high-degree of stability reflects the important functional role of the EC1/2 domains in maintaining an electro-chemical seal at the cell-to-cell junction. Several clusters of water molecules are found buried a sites located both within and between the EC1/2 domains of individual subunits (Fig. 2a, d-f). A cluster of four waters are buried within the EC1/2 domains coordinated by residues D47, E48, Y66, F70 ( $\pi$ bonding), S73, S204/S192, and K209/K197 in Cx50/46 (Fig. 2d). Four additional waters are buried at the lateral EC domain interface formed by neighboring subunits, primarily coordinated by hydrogen-bonding interactions with the peptide backbone and sidechains of Q49, D67, and E208/E196, in Cx50/Cx46 (Fig. 2e). The degree of coordination of these buried waters suggest they contribute to the architectural integrity of EC1/2 docking domains and may in part explain why deleterious mutations at D47, E48, and D67 in Cx50, linked to cataract formation, disrupt junctional coupling and/or biogenesis ${ }^{33-35}$.

The EC1/EC2 domains also play important roles in establishing the specificity of hemi-channel docking interactions formed between different connexin isoforms and the ability to establish the so-called homotypic or heterotypic channels ${ }^{36-38}$. Elucidating the determinants of hemi-channel recognition is therefore critical to understanding the principles dictating cell-type specificity of gap junctional coupling ${ }^{39}$. It has been proposed that isoformspecific hydrogen-bonding patterns that bridge the EC1/EC2 interface govern hemi-channel docking compatibility ${ }^{38,40}$. Contributing to this bridging site in $\mathrm{Cx} 46 / 50$ is a cluster of 12 water molecules (per subunit pair) that are deeply integrated within a dense network of hydrogen bonds between EC1/EC2 residues of opposed subunits (Fig. 2f). At the center of this network is the highly conserved K/R-N-D motif found in EC2 of Group I compatible connexins (including Cx50, Cx46, Cx32, and Cx26). Genetic mutations of this motif in $\mathrm{Cx} 46 / 50$ are linked to congenital cataracts ${ }^{16,41}$, as well as other genetic disorders (e.g., Charcot-Marie-Tooth disease ${ }^{42}$ and non-syndromic deafness ${ }^{43}$ ), when mutated in other Group I connexins. These observations suggest interfacial waters may play previously unappreciated and functionally important roles in establishing the structural integrity of the intercellular channel and contribute to the specificity of hemi-channel docking interactions involved in regulating the formation of intercellular communication pathways.

Long-range ordering of the extracellular lipid leaflet. The degree of long-range stabilization to the local lipid environment observed in the Cx46/50 nanodisc reconstruction is extraordinary, extending several solvent layers away from the protein. DMPC was selected as a model lipid because of the high PC content of mammalian (sheep) lens ${ }^{44}$, and reconstitution studies show DMPC produces Cx46/50 assemblies that are indistinguishable from those formed with native lipids ${ }^{14,45}$. Due to its complete saturation DMPC has a relatively high phase transition (i.e., melting) temperature $\left(T_{\mathrm{m}}\right)$ compared to other biological lipids $\left(T_{\mathrm{m}} \sim 24^{\circ} \mathrm{C}\right.$ in pure lipid vesicles $\left.{ }^{46}\right)$. This value is close to the temperature at which reconstitution was performed $\left(\sim 25^{\circ} \mathrm{C}\right.$, room temperature). However, in nanodiscs the melting temperature of DMPC is reportedly higher $\left(\sim 28^{\circ} \mathrm{C}\right)$, due to compartmentalization effects by the MSP scaffold ${ }^{47}$. Nevertheless, the specific localization of stabilized lipids to the extracellular leaflets observed by CryoEM (and also by MD studies, described below) suggested long-range lipid stabilization is induced through interactions with Cx46/50 (Figs. 1 and 3a).

To gain further insight into the lipid stabilization observed by CryoEM, we analyzed time-averaged densities of DMPC acylchain positions obtained by unbiased all-atom MD simulations for both $\mathrm{Cx} 50$ and $\mathrm{Cx} 46$, conducted at $37^{\circ} \mathrm{C}$, where the starting positions of DMPC molecules had been randomly placed into a $15.4 \times 15.4 \mathrm{~nm}$ lipid bilayer (see "Methods" and Supplementary Fig. 7a). Following equilibration, the resulting acyl-lipid density profiles displayed remarkable similarity to what was resolved by CryoEM (Fig. 3a). In both cases, lipids within the extracellular leaflets are specifically stabilized, as compared to the intracellular lipid leaflet (Fig. 3a). Furthermore, the resolved clusters of acylchain densities obtained by MD display the same hexagonal packing pattern that extends three to four orders beyond the annular shell, as observed by CryoEM (Fig. 3a, inset).

The corroborating results obtained by MD imply that the lipid stabilization observed by CryoEM is directly induced by structural features of the Cx46/50 TM domains and not an artifact of the nanodisc. Each cluster of lipids is bound by a shallow pocket of hydrophobic and aromatic residues, displayed by TM2/3 and TM4 of adjacent subunits (Fig. 3a, b). A cleft, rich 


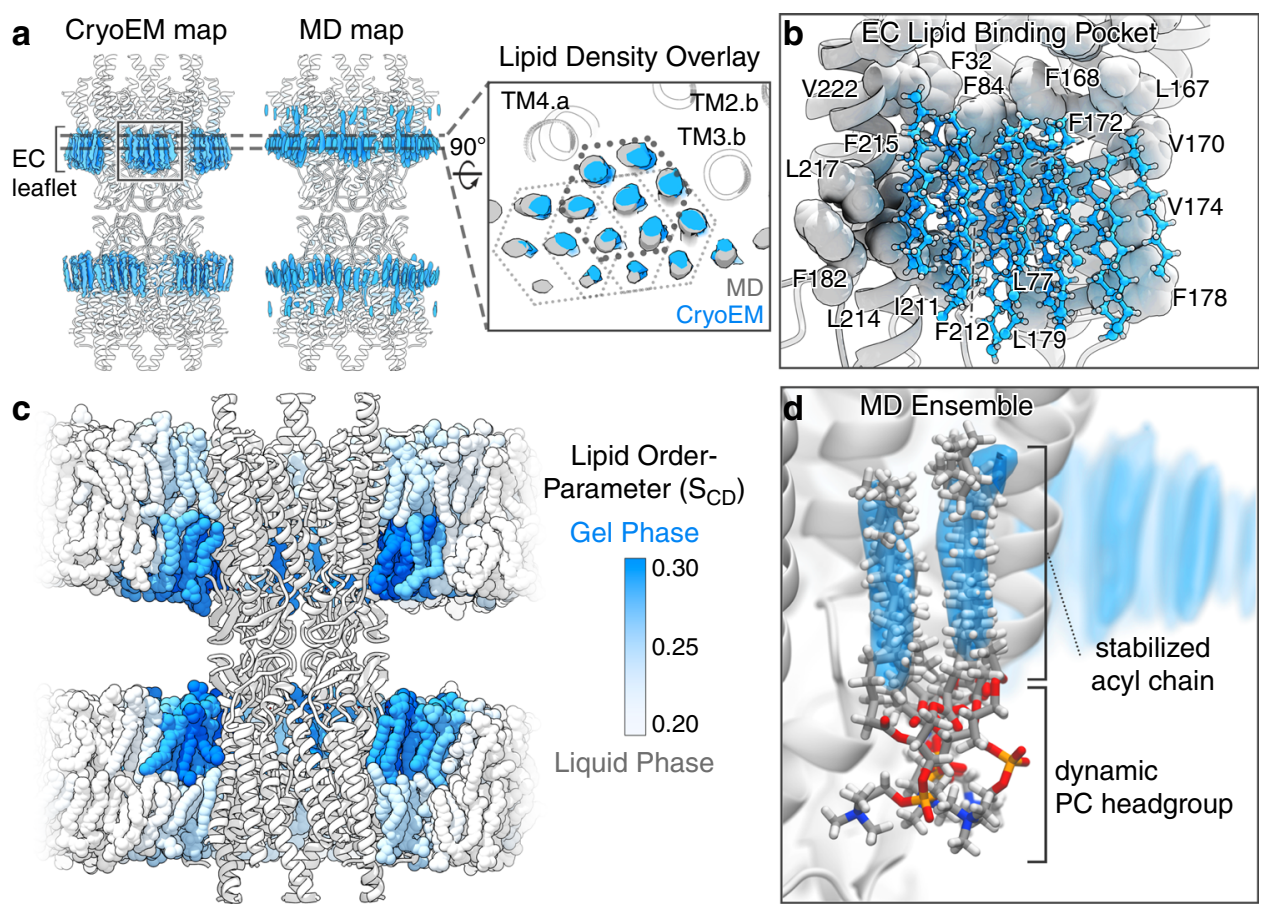

Fig. 3 Cx46/50 induces a local phase separation to the extracellular lipid leaflet. a Comparison of acyl-lipid density maps (blue) obtained by CryoEM and time-averaged all-atom MD simulation, overlaid onto the $C \times 46 / 50$ ribbon structure (white). Inset shows a slice view (rotated $90^{\circ}$ ) of overlaid acyllipid densities by CryoEM (blue) and MD simulation (gray). The hexagonal packing pattern of acyl chains is indicated (solid and dotted lines) and TM helices interacting with lipid are labeled. Different subunits are indicated by suffix ( $a$ or $b$ ). $\mathbf{b}$ Zoom view of the acyl-lipid-binding pocket, with lipid-binding residues displayed (spheres) and labeled (Cx50 numbering). c MD snapshot of Cx50 in phosphatidylcholine (PC) lipid bilayers, with time-averaged lipid order parameter $\left(S_{C D}\right)$ for each lipid indicated by shading (blue $=0.30$ to white $=0.20$ ). $\mathbf{d}$ Zoom view, showing an ensemble super-positioning of symmetry-related lipids obtained by $\mathrm{MD}$ simulation (displayed as all-atom representation, $\mathrm{C}=$ gray, $\mathrm{H}=$ white, $\mathrm{O}=$ red, $\mathrm{N}=$ blue, $\mathrm{P}=$ orange) occupying the MD-based lipid acyl-chain density map (blue).

in aromatic sidechains (formed by F32, F84, L167/L155, and F168/F156 in Cx50/C46), intercalates into the bilayer, appearing to bisect the extracellular leaflet from the more disordered intracellular leaflet (Fig. 3b). In this way, it appears that the acyllipid-binding pocket selectively grasps a large bouquet of lipids from the extracellular leaflet, inducing long-range stabilization to the membrane through extensive Van der Waals interaction.

The extended acyl-lipid chain conformation and hexagonal packing adopted by the bouquet of bound lipids are indicative of a quasi phase transition to the liquid-ordered (or gel-like) state. To obtain a more quantitative assessment of the degree of lipid stabilization, we extracted SN1 and SN2 lipid order parameters $\left(S_{\mathrm{CD}}\right)$ from the MD simulations, which have been parameterized to fit well to experimental NMR-based order parameters ${ }^{48}$. These results are consistent with the notion that lipids in the intracellular leaflet are maintained in a fluid state, whereas Cx46/50 induces a phase transition from a fluid to a gel-like state that is specific to the extracellular lipid leaflet, as indicated by a shift in order parameters to above $\sim 0.25^{49}$ (acyl-chain carbons 4-11; Fig. 3c and Supplementary Fig. 9), which extend $\sim 10-20 \AA$ from the protein surface, as observed by CryoEM.

Although this degree of stabilization to the local lipid environment is likely to depend on lipid type, the general effects may be functionally important. For example, by contributing to the architectural integrity at the gap junctional interface, partitioning specific types of lipids, or even templating longrange hexagonal packing interactions found in plaque assemblies ${ }^{45,50}$. In this context, it is noteworthy that connexins localize to lipid raft domains ${ }^{51,52}$, which are rich in high $T_{\mathrm{m}}$ lipids (e.g., sphingomyelin) and characterized as forming a liquidordered state.
Dynamic lipid configurations captured by CryoEM and MD. Another notable feature of the lipid densities observed in the CryoEM map is that PC head groups are not observed, despite sufficient resolution to expect such features (Figs. 1 and 3a). Super-positioning of representative lipid conformations obtained by $\mathrm{MD}$ show that, although the annular lipid acyl-chains were relatively well ordered and superimpose, their corresponding head groups remain conformationally dynamic and/or heterogeneously positioned (Fig. 3d and Supplementary Movie 4). Such behavior would rationalize the lack of resolvability in the averaged CryoEM density map. In an attempt to resolve this heterogeneity, we conducted $3 \mathrm{D}$ classification analysis on the ensemble CryoEM data ("Methods" and Supplementary Fig. 4), which resulted in three distinct $3 \mathrm{D}$ reconstructions resolved at resolutions of $\sim 2.5 \AA$ (gold-standard FSC) (Fig. $4 \mathrm{a}-\mathrm{c}$ and Supplementary Fig. 4).

In each of the $3 \mathrm{D}$ classes, PC head groups and/or phosphoglycerol backbone of individual annular lipids were uniquely resolved (PC Class 1-3, Fig. 4a). The conformational state of $\mathrm{Cx} 46 / 50$ is very similar in all three classes, and essentially indistinguishable from models derived from the ensemble density map (Ca r.m.s.d.'s = 0.24-0.34 $\AA$ ). Notably, structural features of each of these fully resolved lipids are shared among these classes. For example, the SN2 acyl chain of PC Class 1 overlays with the SN1 chain of PC Class 3 (Fig. 4a, c; yellow and blue). Likewise, the SN1 chain of PC Class 2 also overlays with the SN1 chain of PC Class 3 (Fig. 4a, c; orange and blue). This suggested that multiple, overlapping, configurational states are capable of supporting the same lattice of acyl-chain positions observed in the CryoEM reconstructions, consistent with nonspecific and/or transient binding interactions. 
a
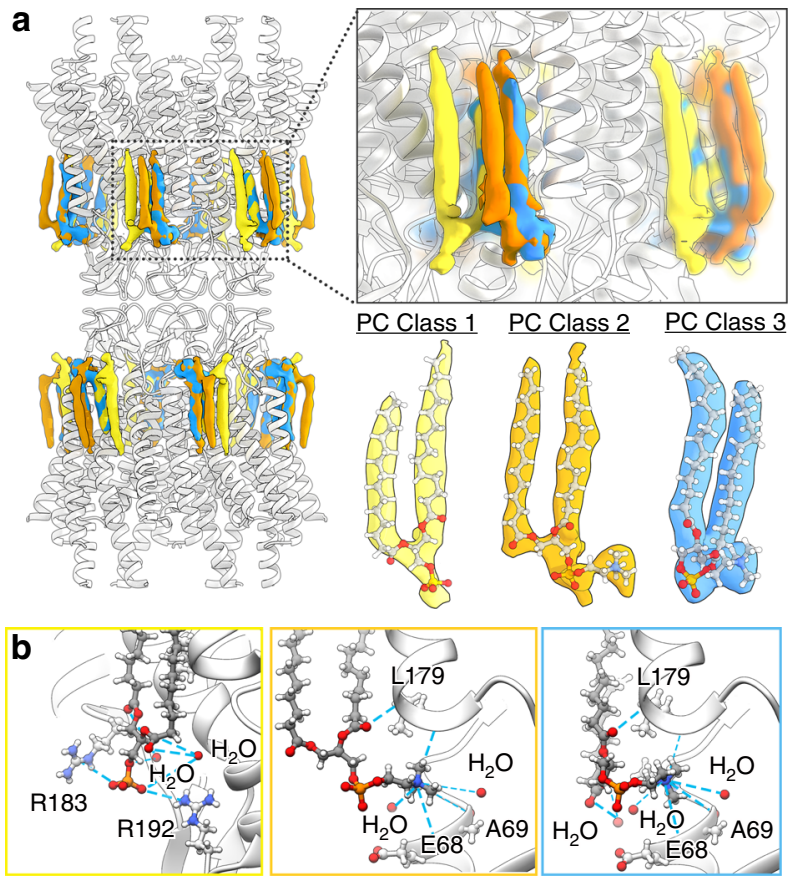
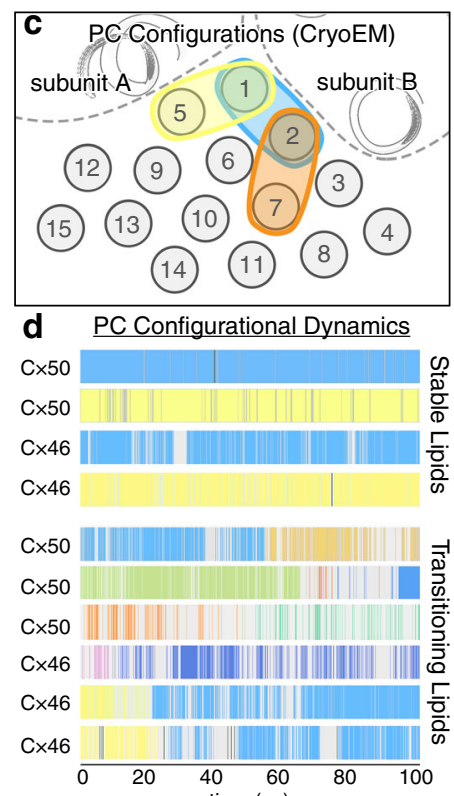

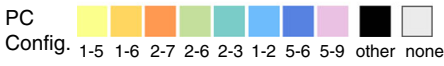

Fig. 4 PC lipid configurational heterogeneity and dynamics resolved by CryoEM and MD. a Segmented phosphatidylcholine (PC) density maps obtained by CryoEM 3D heterogeneity analysis and classification (PC Class 1-yellow, PC Class 2-orange, PC Class 3-blue). Insets show a zoom view displaying the overlapping features of resolved lipid configurations and segmented densities with fitted atomic models obtained from the three PC classes. CryoEM density for all other non-unique acyl-lipid chains, with unresolved head groups, have been omitted for clarity. b Zoom view showing Cx50 hydrogen bond interactions (blue dashed line) between the PC lipid head group and phospho-glycerol backbone. Interacting amino acids and stabilized water molecules are labeled. Yellow box-PC Class 1, orange box-PC Class 2, blue box-PC Class 3. c Illustration showing acyl-chain positions and configurational assignments resolved by CryoEM (represented as gray circles and numbered 1-15). d PC configurational classification and dwell times obtained by all-atom MD simulation, showing representative populations of stable (non-transitioning) and dynamic (transitioning) lipids. PC configurations were classified by acyl-chain occupancy in densities numbered as in $\mathbf{c}$ and colored uniquely (as indicated, bottom of $\mathbf{d}$ ).

The choline head group of PC Class 1 remained unresolved; however, the negatively charged phospho-glycerol backbone is clearly visualized and appears to be stabilized by flanking positively charged arginine residues R183 and R192 in Cx50 (Q171 and R180 in Cx46) (Fig. 4b; left), and hydrogen bonding with two water molecules (part of the belt of extracellular waters described above, see Fig. 2c). PC Class 2 and 3 resolve distinct acyl-chain configurations, yet, both of these states share a similar placement of their positively charged choline head groups. Head group placement of these lipids is supported by nonspecific hydrogen bond interactions with backbone carbonyls presented by EC1 and TM3 (involving residues E68, A69, and L179 in Cx50; position 68 is an $\mathrm{Arg}$ in $\mathrm{Cx} 46$ ) and a buried water molecule (Fig. 4b; center and right). The phospho-glycerol backbones of PC Class $2 / 3$ lipids are coordinated by hydrogen bonding to local waters and the backbone amide of L179 in Cx50 (L167 in Cx46). Remarkably, the SN2 acyl chain and glycerol backbone of the PC Class 2 lipid is completely resolved, despite lacking any direct contact with the Cx46/50 protein interface (Fig. 4a, c).

Collectively, these observations support the notion that although the Cx46/50 acyl-chain interactions appear to be high affinity, the lipid head group interactions are nonspecific and adopt a variety of configurational/conformational states. This is reinforced by our MD simulation studies for both Cx50 and $\mathrm{Cx} 46$, where mapping of $\mathrm{PC}$ arrangements at resolved acyl-chain densities show a variety of configurational states that co-exist within the dodecameric assembly. Furthermore, during the timescale of the simulations (100 ns), time-resolved PC configurations could be classified as being either stable or dynamically transitioning between multiple configurational states (Fig. $4 \mathrm{~d}$ and Supplementary Movie 5, 6). Notably, the most stable (yet overlapping) configurations (e.g., 1-2 and 1-5 configurations) are the same as those resolved by CryoEM 3D classification (Fig. 4a, c, d, blue and yellow, respectively). Yet, other lipid trajectories were observed interconverting between these same configurations over this relatively short timescale (Fig. 4d). The degree of configurational preference diminishes beyond the first two solvent shells, presumably due to the loss of energetic influence induced by protein interactions (Fig. 4d), and reflect the randomized head group arrangements expected of a bulk lipid population. Taken together, these data show $\mathrm{Cx} 46 / 50$ stabilizes the dynamic local lipid environment through nonspecific interactions with the extracellular leaflet, with multiple configurational PC lipid states existing at the annular interface and effectively captured by CryoEM.

\section{Discussion}

The structure and function of membrane proteins are deeply integrated with their lipid environment. Our mechanistic understanding of protein-lipid interactions has been largely shaped by high-resolution structures of membrane proteins where specifically bound lipids have been captured at well-defined binding sites ${ }^{53}$. Yet, most interactions made between membrane proteins and their local membrane environment are relatively nonspecific and highly dynamic. The mechanistic principles and biophysical consequences underlying such interactions remains poorly understood, as these interactions are typically lost during protein purification, or remain too dynamic to resolve by traditional structural methods. By exploiting the potential of lipid nanodisc technologies coupled with single-particle CryoEM and MD simulation, we show that $\mathrm{Cx} 46 / 50$ intercellular 
communication channels form dynamic interactions with annular lipids. These nonspecific interactions have long-range stabilizing effects capable of inducing a phase separation to high $T_{\mathrm{m}}$ lipids, which may extend $\sim 20 \AA$ from the protein surface. These interactions appear selective toward the extracellular leaflet of pure PC membranes, which may have significant consequences on the biomechanical properties and lipid composition of gap junctional domains. In fact, the lack of resolved lipids in the intracellular leaflet may reflect the selectivity at this leaflet toward non-PC lipid types, as suggested for Cx26/32 15 . The methods developed here provide a valuable high-resolution platform for developing our deeper understanding of the specificity and physiological role lipids play in gap junction biology, and how aberrant lipid environments may contribute to connexin-related pathologies. Indeed, the capability of resolving connexin channels beyond the critical threshold of $\sim 2.0-2.5 \AA$ resolution, the precision desired for structure-based drug design-e.g., providing detailed stereochemical models and placement of architectural water molecules -now opens the door to rational development of selective highaffinity pharmacological tools that are desperately needed in this field to better understand and potentially treat a wide range of connexin-opathies ${ }^{54}$.

\section{Methods}

MSP expression and purification. A plasmid containing the coding sequence for MSP1E1 was obtained from Addgene ${ }^{17}$ and the protein was expressed and purified as described 55 , with minor modification. Freshly transformed Escherichia coli cells (BL21Gold-DE3, Agilent, Catalog \#230130) were grown in LB medium containing $50 \mu \mathrm{g} \mathrm{mL}-1$ kanamycin at $37^{\circ} \mathrm{C}$ with shaking (250 r.p.m.). Induction with $0.5 \mathrm{mM}$ isopropyl $\beta$-D-1-thiogalactopyranoside was performed when $\mathrm{OD}_{600}$ reached $0.5-0.6$ and allowed to express for $3-5 \mathrm{~h}$ post induction at $37^{\circ} \mathrm{C}$. Cells were collected by centrifugation at $4000 \times \mathrm{g}$ for $20 \mathrm{~min}$ at $4{ }^{\circ} \mathrm{C}$ and cell pellets were resuspended in MSP Lysis Buffer (40 mM Tris pH 7.4, $1 \%$ Triton X-100, 1 mM phe-

nylmethylsulfonyl fluoride (PMSF)) at a density of $\sim 20 \mathrm{~mL}$ of Lysis Buffer per liter of culture. Cell suspensions were flash frozen in liquid nitrogen and stored at $-86^{\circ} \mathrm{C}$ for up to several months.

Frozen cell suspensions were thawed from $-86^{\circ} \mathrm{C}$ storage, supplemented with $1 \mathrm{mM}$ PMSF, and lysed by sonication on ice. Crude lysate was cleared by ultracentrifugation at $146,550 \times g$ for $30 \mathrm{~min}$ at $4{ }^{\circ} \mathrm{C}$. The supernatant was filtered (Millipore; $0.22 \mu \mathrm{m}$ ) and applied to a gravity column with $5 \mathrm{~mL}$ of HisPur Ni-NTA resin (Thermo Fisher Scientific) prepared in equilibration buffer $(40 \mathrm{mM}$ Tris $\mathrm{pH}$ 7.4). MSP-bound resin was washed with five column volumes (CV) of equilibration buffer, followed by five CVs of each of the following: Triton buffer (40 mM Tris pH 8.0, $300 \mathrm{mM} \mathrm{NaCl}, 1 \%$ TX-100), Cholate buffer (40 mM Tris $\mathrm{pH} 8.0,300 \mathrm{mM} \mathrm{NaCl}, 50 \mathrm{mM}$ cholate), and Imidazole Wash Buffer ( $40 \mathrm{mM}$ Tris pH 8.0, $300 \mathrm{mM} \mathrm{NaCl}, 50 \mathrm{mM}$ imidazole). MSP1E1 was eluted with three CVs of Elution Buffer ( $40 \mathrm{mM}$ Tris pH 8.0, $300 \mathrm{mM} \mathrm{NaCl}, 750 \mathrm{mM}$ imidazole). The eluate was filtered (Millipore; $0.22 \mu \mathrm{m}$ ) and applied to a SEC column (ENC70; BioRad) equilibrated in $20 \mathrm{mM}$ HEPES $\mathrm{pH} 7.4,150 \mathrm{mM} \mathrm{NaCl}$, and $1 \mathrm{mM}$ EDTA by fast protein liquid chromatography (FPLC) (NGC system; BioRad). Peak fractions were monitored by $\mathrm{UV}_{280}$, pooled, and concentrated to $400-600 \mu \mathrm{M}$ using a centrifugal device. Final protein concentration was determined by ultraviolet (UV) absorbance at $280 \mathrm{~nm}$. Samples were aliquoted, flash frozen in liquid nitrogen, and stored at $-86^{\circ} \mathrm{C}$ for up to several months.

Cx46/50 purification and nanodisc reconstitution. Native Cx46/50 intercellular channels were isolated from native lens fiber cells from sheep ${ }^{16}$. Briefly, lamb eyes were obtained from the Wolverine Packers slaughterhouse (Detroit, MI) and the lenses were removed using a surgical blade and stored at $-86^{\circ} \mathrm{C}$. Gap junction intercellular channels were isolated from the core lens fiber tissue, containing $\mathrm{C}$ terminal truncation variants of $\mathrm{Cx} 46$ and $\mathrm{Cx} 50$ (a.k.a. MP38) ${ }^{56-59}$. Details of the purification procedure are provided below.

Lenses were thawed from $-86^{\circ} \mathrm{C}$, core lens fiber cell tissue was dissected from the outer cortical tissue using a surgical blade, and stripped core membranes were prepared as described ${ }^{60-62}$. Total protein concentration was determined by BCA (Pierce) and membranes were stored at $-86^{\circ} \mathrm{C}$, in storage buffer $(10 \mathrm{mM}$ Tris $\mathrm{pH}$ $8.0,2 \mathrm{mM}$ EDTA, $2 \mathrm{mM}$ EGTA) at a total protein concentration of $\sim 2 \mathrm{mg} \mathrm{mL}^{-1}$. Stripped membranes were thawed from $-86^{\circ} \mathrm{C}$ and solubilized with $10 \mathrm{mM}$ Tris pH 8.0, 2 mM EDTA, 2 mM EGTA, and $1 \%\left(\mathrm{wt} \mathrm{vol}^{-1}\right) n$-decyl- $\beta$-D-maltoside (DM) for $30 \mathrm{~min}$ at $37^{\circ} \mathrm{C}$. Insoluble material was cleared by ultra-centrifugation at $146,550 \times g$ for 30 minutes at $4^{\circ} \mathrm{C}$. The supernatant was filtered (Millipore; $0.22 \mu \mathrm{m}$ ) and separated by anion-exchange chromatography (UnoQ, BioRad) with buffer A (10 mM Tris pH 8.0, 2 mM EDTA, 2 mM EGTA, 0.3\% DM (wt vol $\left.{ }^{-1}\right)$ ). Protein was eluted with a $20 \mathrm{CV}$ gradient of buffer B that additionally contained $500 \mathrm{mM} \mathrm{NaCl}$. Elution peaks containing Cx46/50, as determined by
SDS-polyacrylamide gel electrophoresis (PAGE), were pooled and applied to a SEC column (Superose 6 Increase 10/300 GL; GE Healthcare) equilibrated with SEC buffer (20 mM HEPES pH 7.4, $150 \mathrm{mM} \mathrm{NaCl}, 2 \mathrm{mM}$ EDTA, $2 \mathrm{mM}$ EGTA, and $\left.0.3 \% \mathrm{DM}\left(\mathrm{wt} \mathrm{vol}^{-1}\right)\right)$. Peak fractions containing purified $\mathrm{Cx} 46 / 50$ were pooled and concentrated to 5-6 $\mathrm{mg} \mathrm{mL}^{-1}$ with a centrifugal device (Vivaspin 6; $50 \mathrm{kDa}$ cut-off filter; Sartorius). Protein concentration was determined by UV absorbance at $280 \mathrm{~nm}$. All chromatography steps were performed by FPLC at $4{ }^{\circ} \mathrm{C}$.

Freshly purified Cx46/50 was reconstituted into MSP1E1 nanodiscs using DMPC lipids, following established procedures ${ }^{55,63}$. Chloroform-solubilized DMPC (Avanti) was dried under nitrogen gas and left under vacuum overnight to remove residual solvent. The resulting thin film was resuspended in $5 \% \mathrm{DM}\left(\mathrm{wt} \mathrm{vol}^{-1}\right)$ to a final DMPC concentration of $30 \mathrm{mM}$ and solubilized in a sonicator bath at $37^{\circ} \mathrm{C}$. DM-solubilized Cx46/50 (5-6 mg mL $\left.{ }^{-1}\right)$ was combined with DMPC at a molar ratio of 0.6:90 (Cx46/50:DMPC) and incubated at $25^{\circ} \mathrm{C}$ with gentle agitation for $60 \mathrm{~min}$. Purified MSP1E1 was then added at a final molar ratio 0.6:1:90 (Cx46/50: MSP1E1 : DMPC) and allowed to incubate at $25^{\circ} \mathrm{C}$ for an additional $20 \mathrm{~min}$. Detergent was removed with SM-2 Bio-Beads (BioRad) at a ratio of 30:1 beads: detergent $\left(w w^{-1}\right.$ ) by overnight incubation at $25^{\circ} \mathrm{C}$ with gentle agitation. Bio-Beads were removed by filtration and the sample was ultra-centrifuged at $146,550 \times g$ for $15 \mathrm{~min}$ at $4{ }^{\circ} \mathrm{C}$ to remove insoluble material. The supernatant was filtered (Millipore; $0.22 \mu \mathrm{m}$ ) and applied to an SEC column (Superose 6 Increase $10 / 300 \mathrm{GL}$; GE Healthcare) equilibrated in $20 \mathrm{mM}$ HEPES (pH 7.4) and $150 \mathrm{mM}$ $\mathrm{NaCl}$, to separate empty nanodiscs from $\mathrm{Cx} 46 / 50$-embedded nanodiscs. Peak fractions containing both Cx46/50 and MSP1E1, as determined by SDS-PAGE, were collected and concentrated using a centrifugal device (Vivaspin 6; $50 \mathrm{kDa}$ cut-off filter; Sartorius) to a final concentration $\sim 2.5 \mathrm{mg} \mathrm{mL}^{-1}$, as determined by UV absorbance at $280 \mathrm{~nm}$ (Supplementary Fig. 1a). All chromatography steps were performed by FPLC at $4{ }^{\circ} \mathrm{C}$. The presence of both Cx46 and Cx50 in the final sample was confirmed by western blot analysis using polyclonal antibodies directed against the N-terminal domain of $\mathrm{Cx} 46$ (AP11570PU-N, Acris) and the N-terminal domain of Cx50 (LS-C116220, LSBio) (Supplementary Fig. 1b).

Negative-stain electron microscopy. Cx46/50 lipid nanodisc complexes were prepared for negative-stain EM as described ${ }^{16}$. Briefly, a $3 \mu \mathrm{l}$ drop of sample $\left(\sim 0.02 \mathrm{mg} \mathrm{mL}^{-1}\right)$ was applied to a glow-discharged continuous carbon coated EM specimen grid (Ted Pella), blotted with filter paper and washed two times with detergent-free SEC buffer. The specimen was then stained with freshly prepared $0.75 \%\left(\mathrm{wt} \mathrm{vol}^{-1}\right.$ ) uranyl formate (SPI-Chem).

Negatively stained specimens were visualized on a $120 \mathrm{kV}$ TEM (iCorr, Thermo Fisher Scientific) at $\times 49,000$ magnification at the specimen level (Supplementary Fig. 1c). A total of 76 digital micrographs were collected on a $2 \mathrm{k} \times 2 \mathrm{k}$ chargecoupled device camera (Eagle 2 K TEM CCD, Thermo Fisher Scientific) with a calibrated pixel size of $4.37 \AA$ and with nominal defocus values ranging from $1.5-3.0 \mu \mathrm{m}$. All negative-stain image processing was performed in EMAN2.2 $2^{64,65}$ After contrast transfer function (CTF) parameters were determined, micrographs with significant astigmatism or drift were excluded based on visual inspection of Thon rings in the power spectrum. Hand-picked particles (7598) were extracted with $84 \times 84$ pixel box size and subjected to multiple rounds of reference-free twodimensional (2D) classification, resulting in a final dataset of 3826 "good" particles. Representative class averages are shown in Supplementary Fig. 1c, displaying dimensions consistent with the expectation that $\mathrm{Cx} 46 / 50$ intercellular channels had been reconstituted into a pair of lipid nanodiscs.

CryoEM specimen preparation and data collection. Samples were prepared for CryoEM by applying $5 \mu$ freshly purified Cx $46 / 50$ lipid nanodisc complex $\left(\sim 2.5 \mathrm{mg} \mathrm{mL}^{-1}\right)$ to a glow-discharged holey carbon grid (Quantifoil R $1.2 / 1.3$, $400 \mathrm{mesh}$ ) for $10 \mathrm{~s}$. The grid was blotted for $4.0 \mathrm{~s}$ and plunge frozen in liquid ethane using a Vitrobot Mark IV (Thermo Fisher Scientific) at 100\% humidity and stored under liquid nitrogen.

CryoEM specimen grids were imaged on a Titan Krios (Thermo Fisher Scientific) operated at $300 \mathrm{kV}$. Dose-fractionated image stacks were recorded on a Falcon 3EC Direct Electron Detector (Thermo Fisher Scientific) at $\times 120,000$ nominal magnification in counting mode, with a calibrated pixel size of $0.649 \AA \AA_{\text {pixel }}^{-1}$ (Supplementary Fig. 2a). The dose rate was $1.14 \mathrm{e}^{-}$pixel $^{-1} \mathrm{~s}^{-1}$, with five frames per second collected for a total exposure of $30 \mathrm{~s}$, resulting in a total dose for each exposure of $\sim 52.5 \mathrm{e}^{-} \AA^{-2}$. A dataset of 2087 movies was obtained with nominal defocus values ranging from $1.0-2.2 \mu \mathrm{m}$ and data collection parameter were controlled in an automated manner using EPU (Thermo Fisher Scientific).

CryoEM image processing for high-resolution workflow. The full dataset of 2087 movies were corrected for beam-induced motion in RELION-3.066 and CTF estimation was performed with Gctf ${ }^{67}$ on the non-dose-weighted, aligned micrographs. Laplacian-of-Gaussian autopicking in RELION-3.0 yielded an initial set of 756,374 picks, which after multiple rounds of 2D classification left 183,784 bona fide particles (binned to a 64 pixel box, $3.894 \AA_{\text {pixel }}^{-1}$ ). These particles were used to generate a de novo initial model in RELION and subsequent 3D refinement of these particles yielded a map at $8.0 \AA \AA$ resolution (64 pixel box, $3.894 \AA$ pixel $\left.^{-1}\right)$. This map was low-pass-filtered to $20 \AA$ and projected in 14 unique orientations to perform 3D template-based autopicking in RELION-3.0 to yield 1,210,797 particle 
picks. Following multiple rounds of 2D classification, this dataset yielded 379,423 "good" particles (200-pixel box, $1.947 \AA^{\text { pixel }}{ }^{-1}$ ) (Supplementary Fig. 2b). Particles that had been translated within $20 \AA$ of their nearest neighbor were removed to prevent invalidation of gold-standard FSC by duplicate particles. Removal of 120,228 duplicates yielded a 259,195 refined particle set.

This particle set was then re-extracted (1.62 $\AA$ pixel ${ }^{-1}, 280$-pixel box) and subjected to 3D refinement (D6 symmetry), yielding a map at $3.3 \AA$ resolution. A subsequent round of de-duplication ( $20 \AA$ cut-off) yielded 227,618 particles that were again re-extracted $\left(0.974 \AA\right.$ pixel $^{-1}, 512$-pixel box) and subjected to 3D refinement (D6 symmetry), which improved the resolution to $3.2 \AA$. Two rounds of Bayesian polishing and CTF refinement (per-particle defocus, per-micrograph astigmatism) with subsequent 3D refinement (D6 symmetry) yielded a map at $2.7 \AA$ resolution. Particles were then completely unbinned (400-pixel box, $0.649 \AA \mathrm{pixel}^{-1}$ ) and subjected to another round of 3D refinement (D6 symmetry), yielding a map that reached the same resolution prior to unbinning $(2.7 \AA)$. Bayesian polishing and subsequent $3 \mathrm{D}$ refinement of these particles showed no significant improvement. The maps generated up to this stage were obtained without applied masks and there was no evidence of resolved lipids within the intracellular leaflet (see Supplementary Fig. 2, Step 2).

At this stage, the newly developed tools in RELION-3.1-beta ${ }^{68}$ were implemented to estimate the degree of beam tilt and high-order aberrations (threefold and fourfold astigmatism) present in the particle images. Subsequent 3D refinement (D6 symmetry) improved the resolution to $2.2 \AA$ A Particles that had been translated to within $35 \AA$ of their nearest neighbor (6224 particles) were again removed to prevent invalidation of the gold-standard FSC from duplicate particles. The remaining 221,394 particles were subjected to 3D classification into 2 classes with D6 symmetry and a tight solvent mask. Approximately $89 \%$ of the particles $(196,320)$ fell into one class that was subsequently refined to $2.2 \AA$ resolution (D6 symmetry and solvent mask applied). The remaining $11 \%$ of particles $(26,005)$ yielded a $2.0 \AA$ resolution map after 3D refinement (D6 symmetry and solvent mask applied). There was no clear difference in protein conformation between these two classes; however, the high-resolution class was characterized by a particle set with comparatively lower defocus values (mean defocus $=1.20 \pm 0.29 \mu \mathrm{m}(\mathrm{SD})$ ) as compared to the lower resolution class (mean defocus $=1.86 \pm 0.52 \mu \mathrm{m}$ (SD)) (https://github.com/huwjenkins). All subsequent processing steps were performed on this high-resolution particle set.

Particles were re-extracted with an expanded box size (initially to 448 pixels) to mitigate delocalized CTF signal from particle images with relatively high defocus. New polishing parameters were obtained by running the Bayesian polishing job type in RELION-3.1-beta in "Training mode" on a random 5000 particle subset of these refined particles. Bayesian polishing was performed with these new parameters and the subsequent 3D refinement (D6 symmetry and solvent mask applied) improved the resolution slightly to $1.97 \AA$. This process was iterated multiple times with successive increase in box size and incrementally tighter solvent mask applied during Bayesian polishing until no further improvements were observed, resulting in a final box size of 768 pixels. The final map refined to a global 1.90 Å resolution with D6 symmetry and 2.3 Å resolution without symmetry (gold standard, 0.143 cut-off) ${ }^{69}$ (Supplementary Figs. $2 \mathrm{c}$ and $3 \mathrm{a}$ ). The asymmetric reconstruction showed no discernable difference in protein conformation as compared to the D6-symmeterized map. Local resolution of the final map was estimated in RELION-3.1-beta ${ }^{68}$ and local resolution-filtered maps were generated for model building (Supplementary Figs. $2 \mathrm{~d}$ and $3 \mathrm{~b}$ ). A schematic illustrating this high-resolution CryoEM workflow is presented in (Supplementary Fig. 2c).

\section{CryoEM image processing workflow for lipid classification. For classification} and analysis of lipid configurational/conformational heterogeneity, a modified workflow starting from the totally unbinned 227,618 particle set $\left(0.649 \AA\right.$ pixel $^{-1}$, 400-pixel box), which yielded the $2.7 \AA$ resolution map, was applied, as described here (and illustrated in Supplementary Fig. 4a). The particle set was subjected to 3D classification (eight classes), with D6 symmetry and a generous solvent mask applied. Two of the eight classes yielded maps in which the lipid configuration was unambiguously resolved: assigned as PC Class 1 containing 9190 particles ( $\sim \%$ of the data) and PC Class 3 containing 6944 particles ( $\sim 3 \%$ of the data). Overlapping configurations were resolved in two of the other $3 \mathrm{D}$ classes, and so particles from these classes were combined and subjected to a second round of 3D classification with only 2 classes and a tight solvent mask applied. This yielded one class with unresolved lipid configurations and a second class in which the lipid configuration was unambiguously resolved: assigned PC Class 2 containing 6075 particles $(\sim 3 \%$ of the data). Particles assigned to PC Class 1,2, and 3 were separately subjected to a final round of 3D refinement with a solvent mask and D6 symmetry applied (Supplementary Fig. 4a). The final reconstructions from particles in each of these classes all reached $\sim 2.5 \AA$ resolution (gold standard, 0.143 cut-off) (Supplementary Fig. 4b). Local resolution was estimated in RELION-3.1-beta and local resolutionfiltered maps were generated for model building (Supplementary Fig. 4c).

We note that alternative approaches to heterogeneity analysis were also pursued. For example, using the signal-subtraction and symmetry expansion methods in attempt to characterize the specimen heterogeneity at the single subunit level ${ }^{70}$. However, these approaches did not resolve any additional PC lipid conformations/configurations than those captured by the approach described above. It also did not help to resolve the Cx46 and Cx50 isoform heterogeneity.
Atomic modeling, refinement, and validation. For all atomic models of $\mathrm{Cx} 46$ and Cx50, initial models were derived from previously reported CryoEM structure of amphipol-stabilized $\mathrm{Cx} 46$ and $\mathrm{Cx} 50$ (PDB 6MHQ and 6 $\mathrm{MHY}^{16}$, respectively). Initial models were fit as rigid bodies into the D6-symmetrized CryoEM maps with applied local resolution-filtering using UCSF Chimera ${ }^{71}$. All atom models for $\mathrm{Cx} 46$ and $\mathrm{Cx} 50$ were further built into the CryoEM density maps with $\mathrm{COOT}^{72}$ and subjected to real-space refinement in PHENIX ${ }^{73}$ with secondary structure and noncrystallographic symmetry (D6) restraints applied. Several iterations of manual adjustment of the protein model in COOT, followed by real-space refinement in PHENIX, were performed while monitoring model quality with MolProbity ${ }^{74}$ and quality of side chain fit with EMRinger ${ }^{75}$. Coordinate and restraint files for the DMPC ligands were generated with PHENIX eLBOW ${ }^{76}$. DMPC molecules were manually fit into the CryoEM density with COOT. Since density for the PC head groups was not resolved in the high-resolution ensemble CryoEM map $(1.9 \AA$ map), head group, and acyl-chain atoms that could not be accommodated by the density were deleted. For the PC lipid classes $1-3$, the postprocessed maps from RELION were low-pass-filtered to $3.5 \AA$ resolution, to facilitate modeling of the fully resolved PC lipids. COOT was further used to manually place water molecules into solvent densities of the CryoEM maps. Appropriate placement of waters was determined by the following three criteria: (1) confirmation of at least two hydrogen bond donor/acceptor interactions with the FindHBond tool in UCSF Chimera ( $<4 \AA$ donor-acceptor distance) and by visual inspection, (2) confirmation of solvent densities consistently observed in both gold-standard separated halfmaps (contoured $\geq 2.5 \sigma$ ), and (3) as an additional measure we looked for density overlap between the local resolution-filtered CryoEM map (contoured $\geq 5.3 \sigma$ ) and the time-averaged water density map generated by equilibrium MD simulation (contoured $\geq 5.0 \sigma$ ) to help assign weak experimental water densities (Supplementary Fig. 8, see calculation of water density maps from MD described in "Methods" below). However, not all of the assigned CryoEM water densities were observed by MD (76\% of waters were observed at equivalent positions by CryoEM and MD). Several iterations of real-space refinement on the entire model were completed until refinement statistics converged.

Disclosure of unresolved heteromeric assemblies of $\mathrm{Cx} 46 / 50$. All models of Cx46 and Cx50 were built using D6 (12-fold) symmetrized CryoEM maps. As native $\mathrm{Cx} 46 / 50$ intercellular channels may form homomeric and/or various patterns of heteromeric/heterotypic configurations ${ }^{16,77,78}$, this map most likely represent a heterogeneous mixture of these two isoforms ${ }^{16}$. This approach was chosen, because all attempts to separate the heteromeric/heterotypic assembly of these two isoforms using image classification procedures were unsuccessful (presumably due to the close sequence and structural similarity of these two isoforms) (see also ref. ${ }^{16}$ ). Indeed, Cx46 and Cx50 are 80\% identical and 89\% similar in sequence over the resolved structural domains, whereas sites of difference are typically at solvent-exposed regions (Supplementary Fig. 6a). Despite this limitation, all atomic models generated by this approach showed good stereochemical refinement statistics (see Supplementary Table 1) and significant improvements to the previously described amphipol-stabilized models that were refined to $3.4 \AA$ resolution (Supplementary Fig. 5a-e). It is important to note that sites in the density maps where the sequence of $\mathrm{Cx} 46$ and $\mathrm{Cx} 50$ are identical or similar, both models fit well into the D6-symmetrized map and these regions tend to display well-resolved side chain density (Supplementary Fig. 3c, d). Over regions where the sequence of $\mathrm{Cx} 46$ and $\mathrm{Cx} 50$ differ, side chain density is sometimes weaker and/or displays appearance of density consistent with a mixture of both isoforms (Supplementary Fig. 3c, d). These observations are possibly due to the imposed D6 symmetry averaging of density belonging to two different sidechains in these areas or relative flexibility at these sites, as many of these residues are also solvent exposed. In these areas of difference, where EM density is observed, both Cx46 and Cx50 can be fit into the density equally well (Supplementary Fig. 3c, d). Nevertheless, caution should be used with interpretation of the conformational details at these sites of isoform difference.

Molecular dynamics simulations. Visual MD (VMD) v1.9.379 was used to build systems for sheep Cx46 and Cx50 in a dual lipid bilayer with varying salt conditions, designed to mimic either the cellular environment (cytoplasmic $\mathrm{KCl}$, extracellular $\mathrm{NaCl}$ ) or experimental CryoEM conditions (uniform $\mathrm{NaCl}$ ). To produce unbiased analysis of water and lipid interactions, all water and lipid molecules derived by CryoEM analysis were removed from the Cx46 and Cx50 models prior to the MD setup. Each system comprised the full dodecameric gap junction intercellular channel, prepared in explicit water (model TIP3P) and embedded in two lipid bilayers composed of DMPC, mimicking the cell-to-cell junction. For all models, sidechains were protonated according to neutral conditions and the HSD model was used for all histidine residues. Disulfide bonds identified in the experimental structures were enforced. Amino acids corresponding to the ICL (residues 110-136 in sheep Cx46 and residues 110-148 in sheep Cx50) and CTD (residues 225-413 in sheep Cx46 and residues 237-440 in sheep Cx50) were not included for the MD simulations, as experimental data describing the structure of these large flexible domains ( $\sim 30$ residue ICL and $\sim 200$ residue CTD in Cx46 and Cx50) are missing. The introduced $\mathrm{N}$ - and C-terminal residues resulting from the missing ICL segment (sheep Cx46 R109 and K137; sheep Cx50 R109 and R149) were neutralized. All of the systems were modified with an N-terminal acetylation 
(at the starting residue Gly 2) in VMD through an all-atom acetylation patch in the automated PSF-Builder, in accordance with previously described proteomics analysis on native $\mathrm{Cx} 46 / 50^{16,59,80}$ and the expectation that this species would predominate in cells ${ }^{81}$. A complete list of modeled residues for each system is provided in Supplementary Fig. 7a.

The prepared protein structures were submerged in a hydration shell using Solvate 1.0.1 ${ }^{82}$. Water was removed from sections of the channel corresponding to transmembrane domains, based on hydrophobic character and localization of lipid nanodisc observed in the experimental CryoEM data $( \pm 20-50 \AA$ from the center of the channel). The CHARMM-GUI membrane builder ${ }^{83}$ was used to build the DMPC bilayers (pre-melted), with dimensions of $154 \times 154 \AA$ for $\mathrm{Cx} 46$ and $\mathrm{Cx} 50$, and lipids overlapping with protein were removed. The entire system was then placed in a water box with dimensions $147 \times 147 \times 174 \AA$ for both $\mathrm{Cx} 46$ and $\mathrm{Cx} 50$, using VMD's Solvate plugin. The system was neutralized using the Autoionize plugin, then $150 \mathrm{mM} \mathrm{KCl}$ and $150 \mathrm{mM} \mathrm{NaCl}$ was added to the solvent areas corresponding to intracellular and extracellular regions of the simulation box for the "KCl" systems, whereas the "NaCl" systems contained $150 \mathrm{mM} \mathrm{NaCl}$ for the entire box. A summary of atoms counts for each system is provided in Supplementary Fig. 7a.

Graphics processing unit (GPU)-accelerated nanoscale MD (NAMD) $2.13^{84}$ was used for all classical MD simulations, using the CHARMM36 force field ${ }^{85}$ for all atoms and TIP3P explicit model for water. Each system was prepared following the same minimization and equilibration protocol as follows. An initial minimization step, where the lipids, solvent, and ions were allowed to minimize around the protein, was performed, with the protein harmonically constrained for $1 \mathrm{~ns}$, with $1 \mathrm{fs}$ timestep and constant number of particles, volume and temperature $(310 \mathrm{~K})$ (NVT ensemble). A second minimization step was applied, where the system was free to minimize with a harmonic constraint on the protein backbone to ensure stable quaternary structure for $1 \mathrm{~ns}$-lipids relax and compress during minimization steps with minimized dimensions equal to the water box $(14.7 \times$ 14.7). The entire system was then released from restraints and subjected to all-atom equilibration runs employing Langevin thermostat, with a constant number of particles, pressure ( $1 \mathrm{~atm})$ and temperature $(310 \mathrm{~K})$ (NPT ensemble), with $2 \mathrm{fs}$ time steps and allowed to proceed for $30 \mathrm{~ns}$. Periodic boundary conditions were used to allow for the smoothed particle mesh Ewald calculation of electrostatics. Finally, two independent $100 \mathrm{~ns}$ production runs were seeded with randomly initialized velocities from the initial equilibration simulation-providing $200 \mathrm{~ns}$ of production for each system. r.m.s.d. and r.m.s.f. were calculated using VMD, and r.m.s.f. values were displayed to the protein structure using UCSF Chimera (Supplementary Fig. 7b-d). All systems approached a steady r.m.s.d. within $30 \mathrm{~ns}$ of the equilibration phase (Supplementary Fig. 7b) and r.m.s.f. values appeared wellbehaved over the production periods, including regions corresponding to the NTH domain $^{16}$ (Supplementary Fig. 7c, d). The only significant fluctuations (i.e., $>2.5 \AA$ ) occurred at the TM2, TM3, and TM4 cytoplasmic termini, which is expected, as these regions form the boundary to the intrinsically disordered ICL and CTD regions of the protein (not modeled). All systems maintained an electro-chemical seal to extracellular sodium ions $\left(\mathrm{Na}^{+}\right)$around the extracellular docking domains during MD simulation.

Calculation of MD-based density maps. The Volmap plugin in VMD was used for the calculation of volumetric density maps, by replacing each atom with a normalized Gaussian distribution, whose standard deviation is equal to the radius of the atom. All of the Gaussians are summed and distributed on a grid for each frame of the simulation. The grids were re-sampled to a final voxel resolution of $0.649 \AA$ $\AA$ to match the pixel size used in the CryoEM reconstruction. Water, ion, and lipid maps were calculated from each of two 100 ns production runs and subsequently averaged and symmetrized (D6 symmetry) with the relion_image_handler tool in RELION-3.066. Lipid and water density maps produced from Cx46 and Cx50 MD simulations contained significant overlap to each other and to the CryoEM maps; however, the maps produced from Cx50 MD simulations were of higher quality and were selected for detailed comparative analysis to the CryoEM density maps (Fig. 3 and Supplementary Fig. 8). Ion density maps showed only a few features and did not correspond to densities observed by CryoEM and were therefore excluded from further analysis.

Area per lipid and lipid order parameter calculations. Area per lipid for each membrane, separated by intracellular and extracellular leaflet, were calculated using the program FATSLiM ${ }^{86}$, and were used as an indicator of equilibration of the lipid systems (Supplementary Fig. 9a).

$$
S_{\mathrm{CD}} \equiv-\left\langle\frac{3 \cos ^{2}\left(\theta_{\mathrm{CD}}\right)-1}{2}\right\rangle
$$

The $S_{\mathrm{CD}}$ lipid order parameter, as defined by Eq. (1), measures the orientation of the SN1 and SN2 acyl chains by monitoring the angle that each acyl C-H vector makes with the bilayer normal $\theta_{\mathrm{CD}}$. The calculations of $S_{\mathrm{CD}}$ were done using the VMD script calc_op.tcl ${ }^{87}$. To analyze the distance dependence of $S_{\mathrm{CD}}$ in the respective membrane leaflets, the averaged $S_{\mathrm{CD}}$ values were calculated in $5 \AA$ concentric shells around the protein (SN1 and SN2 calculated, separately). $S_{\mathrm{CD}}$ of lipids from both membranes are averaged together, whereas the intra- and extracellular leaflets were averaged separately (Supplementary Fig. 9b-e). To visualize the order parameter mapped to the structure, the time-averaged $S_{\mathrm{CD}}$ values were calculated for each lipid (SN1 and SN2 combined values for acyl carbons 4-11) and colored according to this value using UCSF Chimera (Fig. 3c).

MD lipid configuration analysis. Analysis of PC lipid configuration (i.e., acylchain positioning) was performed using in-house scripts to assess how phospholipids are organized within the extracellular leaflet of the $\mathrm{Cx} 46$ and $\mathrm{Cx} 50$ intercellular channels during MD simulation, as compared to the PC configurations classified by CryoEM. This was done by counting the instances of a single DMPC molecule occupying the region bounded by the MD-based lipid density, contoured at $\sigma_{\min }=8$ (Fig. 3a). The lipid acyl-chain density maps calculated from the Cx50 MD simulations reveal more than 19 resolved rods $(i)$ of density per connexin subunit (12 subunits) and each rod was arbitrarily numbered 1 through 19 (total of 228 acyl-chain positions). A lipid was classified in a state when both acyl chains occupied a density, state $\equiv$ " $i-j$ " (where $i \neq j$ ). A rod density is considered occupied if at least five carbons of a lipid's acyl chain are within the density, such that $\sigma_{\text {carbon }} \geq \sigma_{\text {min }}$. This classification scheme was applied to every lipid within $15 \AA$ of the protein, over each frame (0.1 ns per frame). To analyze the dynamics of lipids surrounding the protein, the state of each lipid (e.g., "1-2," "1-5," "none," etc.) was monitored and recorded at every frame providing a time series of lipid configurational dynamics in state space (Fig. 4d)

Statistical analysis. Ninety-five percent confidence intervals for Ca r.m.s.f. values are reported $(n=24)$ using a two-tailed Student's $t$-test. FSC was performed using gold standard methods with a 0.143 cut-off criteria ${ }^{69}$. No statistical methods were used to predetermine sample size for the CryoEM dataset. The experiments were not randomized and investigators were not blinded to allocation during experiments and outcome assessment.

Figures and movie preparation. Figure panels and movies were created using Chimera $^{71}$, ChimeraX ${ }^{88}, \mathrm{VMD}^{79}$, and Blender ${ }^{89}$. Figures were composited in Adobe Photoshop. Movies were composited in Blender.

Reporting summary. Further information on research design is available in the Nature Research Reporting Summary linked to this article.

\section{Data availability}

Data supporting the findings of this study are available from the corresponding author upon reasonable request. A reporting summary for this Article is available as a Supplementary Information file. CryoEM density maps, including half-maps, pre- and postprocessed maps, and masks have been deposited to the Electron Microscopy Data Bank (EMD-22358, EMD-22382, EMD-22390, and EMD-22391). Coordinates for Cx50 and Cx46 atomic models have been deposited to the Protein Data Bank: PDB7JJP and PDB7JKC correspond to the high-resolution models, PDB7JLW and PDB7JMD correspond to the models from PC Class 1, PDB7JM9 and PDB7JN0 correspond to PC Class 2, and PDB7JMC and PDB7JN1 correspond to PC Class 3. The original multiframe micrographs have been deposited to EMPIAR (EMPIAR-10480). MD trajectory files and MD-based density maps have been deposited to Zenodo (https://doi.org/ 10.5281/zenodo.3951861).

\section{Code availability}

In-house code developed for the analysis of MD trajectories to assess lipid configurational state dynamics have been deposited on the Reichow Lab GitHub repository (https://github.com/reichow-lab/Flores-Haddad-Dolan_et-al. NatCommun2020) and Zenodo (https://doi.org/10.5281/zenodo.3955131).

Received: 30 June 2020; Accepted: 7 August 2020; Published online: 28 August 2020

\section{References}

1. Goodenough, D. A. \& Paul, D. L. Gap junctions. Cold Spring Harb. Perspect Biol. 1, a002576 (2009).

2. Sohl, G. \& Willecke, K. Gap junctions and the connexin protein family. Cardiovasc. Res. 62, 228-232 (2004).

3. Harris, A. L. Connexin channel permeability to cytoplasmic molecules. Prog Biophys. Mol. Biol. 94, 120-143 (2007).

4. Bonacquisti, E. E. \& Nguyen, J. Connexin $43(\mathrm{Cx} 43)$ in cancer: Implications for therapeutic approaches via gap junctions. Cancer Lett. 442, 439-444 (2019).

5. Delmar, M. et al. Connexins and disease. Cold Spring Harb. Perspect. Biol. https://doi.org/10.1101/cshperspect.a029348 (2017).

6. Garcia, I. E. et al. Connexinopathies: a structural and functional glimpse. BMC Cell Biol. 17(Suppl 1), 17 (2016). 
7. Aasen, T., Mesnil, M., Naus, C. C., Lampe, P. D. \& Laird, D. W. Gap junctions and cancer: communicating for 50 years. Nat. Rev. Cancer 16, 775-788 (2016).

8. Makowski, L., Caspar, D. L., Phillips, W. C. \& Goodenough, D. A. Gap junction structures. II. Analysis of the X-ray diffraction data. J. Cell Biol. 74, 629-645 (1977).

9. Revel, J. P. \& Karnovsky, M. J. Hexagonal array of subunits in intercellular junctions of the mouse heart and liver. J. Cell Biol. 33, C7-C12 (1967).

10. Sosinsky, G. E. \& Nicholson, B. J. Structural organization of gap junction channels. Biochim. Biophys. Acta 1711, 99-125 (2005).

11. Malewicz, B., Kumar, V. V., Johnson, R. G. \& Baumann, W. J. Lipids in gap junction assembly and function. Lipids 25, 419-427 (1990).

12. Cascio, M. Connexins and their environment: effects of lipids composition on ion channels. Biochim. Biophys. Acta 1711, 142-153 (2005).

13. Puebla, C., Retamal, M. A., Acuna, R. \& Saez, J. C. Regulation of connexinbased channels by fatty acids. Front. Physiol. 8, 11 (2017).

14. Kistler, J., Goldie, K., Donaldson, P. \& Engel, A. Reconstitution of native-type noncrystalline lens fiber gap junctions from isolated hemichannels. J. Cell Biol. 126, 1047-1058 (1994).

15. Locke, D. \& Harris, A. L. Connexin channels and phospholipids: association and modulation. BMC Biol. 7, 52 (2009).

16. Myers, J. B. et al. Structure of native lens connexin $46 / 50$ intercellular channels by cryo-EM. Nature 564, 372-377 (2018).

17. Denisov, I. G., Grinkova, Y. V., Lazarides, A. A. \& Sligar, S. G. Directed selfassembly of monodisperse phospholipid bilayer nanodiscs with controlled size. J. Am. Chem. Soc. 126, 3477-3487 (2004).

18. Maeda, S. et al. Structure of the connexin 26 gap junction channel at $3.5 \mathrm{~A}$ resolution. Nature 458, 597-602 (2009).

19. Bennett, B. C. et al. An electrostatic mechanism for $\mathrm{Ca}(2+)$-mediated regulation of gap junction channels. Nat. Commun. 7, 8770 (2016).

20. Gong, X. Q. \& Nicholson, B. J. Size selectivity between gap junction channels composed of different connexins. Cell Commun. Adhes. 8, 187-192 (2001).

21. Trexler, E. B., Bukauskas, F. F., Kronengold, J., Bargiello, T. A. \& Verselis, V. K. The first extracellular loop domain is a major determinant of charge selectivity in connexin46 channels. Biophys. J. 79, 3036-3051 (2000).

22. Kronengold, J., Trexler, E. B., Bukauskas, F. F., Bargiello, T. A. \& Verselis, V. K. Pore-lining residues identified by single channel SCAM studies in Cx46 hemichannels. Cell Commun. Adhes. 10, 193-199 (2003).

23. Verselis, V. K., Trelles, M. P., Rubinos, C., Bargiello, T. A. \& Srinivas, M. Loop gating of connexin hemichannels involves movement of pore-lining residues in the first extracellular loop domain. J. Biol. Chem. 284, 4484-4493 (2009).

24. Oh, S., Verselis, V. K. \& Bargiello, T. A. Charges dispersed over the permeation pathway determine the charge selectivity and conductance of a Cx32 chimeric hemichannel. J. Physiol. 586, 2445-2461 (2008).

25. Kwon, T. et al. Molecular dynamics simulations of the Cx26 hemichannel: insights into voltage-dependent loop-gating. Biophys. J. 102, 1341-1351 (2012).

26. Kwon, T., Harris, A. L., Rossi, A. \& Bargiello, T. A. Molecular dynamics simulations of the Cx26 hemichannel: evaluation of structural models with Brownian dynamics. J. Gen. Physiol. 138, 475-493 (2011).

27. Zonta, F., Polles, G., Zanotti, G. \& Mammano, F. Permeation pathway of homomeric connexin 26 and connexin 30 channels investigated by molecular dynamics. J. Biomol. Struct. Dyn. 29, 985-998 (2012).

28. Bargiello, T. A., Tang, Q., Oh, S. \& Kwon, T. Voltage-dependent conformational changes in connexin channels. Biochim. Biophys. Acta 1818, 1807-1822 (2012).

29. Tong, X. et al. The first extracellular domain plays an important role in unitary channel conductance of Cx50 gap junction channels. PLoS ONE 10, e0143876 (2015)

30. Lopez, W. et al. Mechanism of gating by calcium in connexin hemichannels. Proc. Natl Acad. Sci. USA 113, E7986-E7995 (2016).

31. Garcia, I. E. et al. The syndromic deafness mutation G12R impairs fast and slow gating in Cx26 hemichannels. J. Gen. Physiol. 150, 697-711 (2018).

32. Rubinos, C., Sanchez, H. A., Verselis, V. K. \& Srinivas, M. Mechanism of inhibition of connexin channels by the quinine derivative $\mathrm{N}$-benzylquininium. J. Gen. Physiol. 139, 69-82 (2012).

33. Banks, E. A. et al. Connexin mutation that causes dominant congenital cataracts inhibits gap junctions, but not hemichannels, in a dominant negative manner. J. Cell Sci. 122, 378-388 (2009).

34. Berthoud, V. M. et al. Connexin50D47A decreases levels of fiber cell connexins and impairs lens fiber cell differentiation. Invest. Ophthalmol. Vis. Sci. 54, 7614-7622 (2013).

35. Reis, L. M. et al. Whole exome sequencing in dominant cataract identifies a new causative factor, CRYBA2, and a variety of novel alleles in known genes. Hum. Genet. 132, 761-770 (2013).

36. White, T. W., Bruzzone, R., Wolfram, S., Paul, D. L. \& Goodenough, D. A. Selective interactions among the multiple connexin proteins expressed in the vertebrate lens: the second extracellular domain is a determinant of compatibility between connexins. J. Cell Biol. 125, 879-892 (1994).
37. White, T. W., Paul, D. L., Goodenough, D. A. \& Bruzzone, R. Functional analysis of selective interactions among rodent connexins. Mol. Biol. Cell 6, 459-470 (1995)

38. Nakagawa, S. et al. Asparagine 175 of connexin 32 is a critical residue for docking and forming functional heterotypic gap junction channels with connexin26. J. Biol. Chem. 286, 19672-19681 (2011).

39. Cottrell, G. T. \& Burt, J. M. Functional consequences of heterogeneous gap junction channel formation and its influence in health and disease. Biochim. Biophys. Acta 1711, 126-141 (2005).

40. Bai, D. \& Wang, A. H. Extracellular domains play different roles in gap junction formation and docking compatibility. Biochem. J. 458, 1-10 (2014).

41. Schadzek, P. et al. The cataract related mutation N188T in human connexin 46 (hCx46) revealed a critical role for residue N188 in the docking process of gap junction channels. Biochim. Biophys. Acta 1858, 57-66 (2016).

42. Silander, K. et al. Spectrum of mutations in Finnish patients with CharcotMarie-Tooth disease and related neuropathies. Hum. Mutat. 12, 59-68 (1998)

43. Primignani, P. et al. A novel dominant missense mutation-D179N-in the GJB2 gene (Connexin 26) associated with non-syndromic hearing loss. Clin. Genet. 63, 516-521 (2003).

44. Deeley, J. M. et al. Human lens lipids differ markedly from those of commonly used experimental animals. Biochim. Biophys. Acta 1781, 288-298 (2008).

45. Lampe, P. D. et al. In vitro assembly of gap junctions. J. Struct. Biol. 107, 281-290 (1991)

46. Mabrey, S. \& Sturtevant, J. M. Investigation of phase transitions of lipids and lipid mixtures by sensitivity differential scanning calorimetry. Proc. Natl Acad. Sci. USA 73, 3862-3866 (1976).

47. Shaw, A. W., McLean, M. A. \& Sligar, S. G. Phospholipid phase transitions in homogeneous nanometer scale bilayer discs. FEBS Lett. 556, 260-264 (2004).

48. Vermeer, L. S., de Groot, B. L., Reat, V., Milon, A. \& Czaplicki, J. Acyl chain order parameter profiles in phospholipid bilayers: computation from molecular dynamics simulations and comparison with $2 \mathrm{H}$ NMR experiments. Eur. Biophys. J. 36, 919-931 (2007).

49. Khakbaz, P. \& Klauda, J. B. Investigation of phase transitions of saturated phosphocholine lipid bilayers via molecular dynamics simulations. Biochim. Biophys. Acta Biomembr. 1860, 1489-1501 (2018).

50. Caspar, D. L., Goodenough, D. A., Makowski, L. \& Phillips, W. C. Gap junction structures. I. Correlated electron microscopy and x-ray diffraction. J. Cell Biol. 74, 605-628 (1977).

51. Schubert, A. L., Schubert, W., Spray, D. C. \& Lisanti, M. P. Connexin family members target to lipid raft domains and interact with caveolin-1. Biochemistry 41, 5754-5764 (2002).

52. Locke, D., Liu, J. \& Harris, A. L. Lipid rafts prepared by different methods contain different connexin channels, but gap junctions are not lipid rafts. Biochemistry 44, 13027-13042 (2005).

53. Hunte, C. Specific protein-lipid interactions in membrane proteins. Biochem. Soc. Trans. 33, 938-942 (2005).

54. Spray, D. C., Rozental, R. \& Srinivas, M. Prospects for rational development of pharmacological gap junction channel blockers. Curr. Drug Targets 3 , 455-464 (2002).

55. Ritchie, T. K. et al. Chapter 11 - Reconstitution of membrane proteins in phospholipid bilayer nanodiscs. Methods Enzymol. 464, 211-231 (2009).

56. Kistler, J., Christie, D. \& Bullivant, S. Homologies between gap junction proteins in lens, heart and liver. Nature 331, 721-723 (1988).

57. Kistler, J., Schaller, J. \& Sigrist, H. MP38 contains the membrane-embedded domain of the lens fiber gap junction protein MP70. J. Biol. Chem. 265, 13357-13361 (1990).

58. White, T. W., Bruzzone, R., Goodenough, D. A. \& Paul, D. L. Mouse Cx50, a functional member of the connexin family of gap junction proteins, is the lens fiber protein MP70. Mol. Biol. Cell 3, 711-720 (1992).

59. Wang, Z. \& Schey, K. L. Phosphorylation and truncation sites of bovine lens connexin 46 and connexin 50. Exp. Eye Res. 89, 898-904 (2009).

60. Reichow, S. L. et al. Allosteric mechanism of water-channel gating by Ca2+-calmodulin. Nat. Struct. Mol. Biol. 20, 1085-1092 (2013).

61. Gold, M. G. et al. AKAP2 anchors PKA with aquaporin-0 to support ocular lens transparency. EMBO Mol. Med. 4, 15-26 (2012).

62. Reichow, S. L. \& Gonen, T. Noncanonical binding of calmodulin to aquaporin-0: implications for channel regulation. Structure 16, 1389-1398 (2008).

63. Efremov, R. G., Gatsogiannis, C. \& Raunser, S. Lipid nanodiscs as a tool for high-resolution structure determination of membrane proteins by singleparticle cryo-EM. Methods Enzymol. 594, 1-30 (2017).

64. Tang, G. et al. EMAN2: an extensible image processing suite for electron microscopy. J. Struct. Biol. 157, 38-46 (2007).

65. Ludtke, S. J. Single-particle refinement and variability analysis in EMAN2.1. Methods Enzymol. 579, 159-189 (2016).

66. Zivanov, J. et al. New tools for automated high-resolution cryo-EM structure determination in RELION-3. eLife 7, https://doi.org/10.7554/eLife.42166 (2018). 
67. Zhang, K. Gctf: Real-time CTF determination and correction. J. Struct. Biol. 193, 1-12 (2016)

68. Zivanov, J., Nakane, T. \& Scheres, S. H. W. Estimation of high-order aberrations and anisotropic magnification from cryo-EM data sets in RELION-3.1. IUCrJ 7, 253-267 (2020).

69. Scheres, S. H. \& Chen, S. Prevention of overfitting in cryo-EM structure determination. Nat. Methods 9, 853-854 (2012).

70. Scheres, S. H. Processing of structurally heterogeneous cryo-EM data in RELION. Methods Enzymol. 579, 125-157 (2016).

71. Pettersen, E. F. et al. UCSF Chimera-a visualization system for exploratory research and analysis. J. Comput. Chem. 25, 1605-1612 (2004).

72. Emsley, P., Lohkamp, B., Scott, W. G. \& Cowtan, K. Features and development of Coot. Acta Crystallogr. D66, 486-501 (2010).

73. Afonine, P. V. et al. Real-space refinement in PHENIX for cryo-EM and crystallography. Acta Crystallogr. D. Struct. Biol. 74, 531-544 (2018).

74. Williams, C. J. et al. MolProbity: more and better reference data for improved all-atom structure validation. Protein Sci. 27, 293-315 (2018).

75. Barad, B. A. et al. EMRinger: side chain-directed model and map validation for 3D cryo-electron microscopy. Nat. Methods 12, 943-946 (2015).

76. Moriarty, N. W., Grosse-Kunstleve, R. W. \& Adams, P. D. Electronic Ligand Builder and Optimization Workbench (eLBOW): a tool for ligand coordinate and restraint generation. Acta Crystallogr. D. Biol. Crystallogr. 65, 1074-1080 (2009).

77. Konig, N. \& Zampighi, G. A. Purification of bovine lens cell-to-cell channels composed of connexin44 and connexin50. J. Cell Sci. 108(Pt 9), 3091-3098 (1995).

78. Jiang, J. X. \& Goodenough, D. A. Heteromeric connexons in lens gap junction channels. Proc. Natl Acad. Sci. USA 93, 1287-1291 (1996).

79. Humphrey, W., Dalke, A. \& Schulten, K. VMD: visual molecular dynamics. J. Mol. Graph. 14, 33-38, 27-38. (1996).

80. Shearer, D., Ens, W., Standing, K. \& Valdimarsson, G. Posttranslational modifications in lens fiber connexins identified by off-line-HPLC MALDIquadrupole time-of-flight mass spectrometry. Invest. Ophthalmol. Vis. Sci. 49, 1553-1562 (2008).

81. Varland, S., Osberg, C. \& Arnesen, T. N-terminal modifications of cellular proteins: the enzymes involved, their substrate specificities and biological effects. Proteomics 15, 2385-2401 (2015).

82. Grubmuller, H., Heymann, B. \& Tavan, P. Ligand binding: molecular mechanics calculation of the streptavidin-biotin rupture force. Science 271, 997-999 (1996).

83. Wu, E. L. et al. CHARMM-GUI Membrane Builder toward realistic biological membrane simulations. J. Comput. Chem. 35, 1997-2004 (2014).

84. Phillips, J. C. et al. Scalable molecular dynamics with NAMD. J. Comput. Chem. 26, 1781-1802 (2005).

85. Huang, J. \& MacKerell, A. D. Jr CHARMM36 all-atom additive protein force field: validation based on comparison to NMR data. J. Comput. Chem. 34, 2135-2145 (2013).

86. Buchoux, S. FATSLiM: a fast and robust software to analyze MD simulations of membranes. Bioinformatics 33, 133-134 (2017).

87. Piggot, T. J., Allison, J. R., Sessions, R. B. \& Essex, J. W. On the calculation of acyl chain order parameters from lipid simulations. J. Chem. Theory Comput. 13, 5683-5696 (2017).

88. Goddard, T. D. et al. UCSF ChimeraX: meeting modern challenges in visualization and analysis. Protein Sci. 27, 14-25 (2018).

89. Community, B. O. Blender-a 3D modelling and rendering package. http:// www.blender.org (Stichting Blender Foundation, 2018).

\section{Acknowledgements}

We thank Dror Chorev and Carol V. Robinson for help analyzing specimens prepared for CryoEM. We are grateful to the staff at the OHSU Multiscale Microscopy Core and Advanced Computing center, and to the Pacific Northwest Center for CryoEM (supported by NIH Grant U24GM129547), and accessed through EMSL (grid.436923.9). J.A.F. and B. G.H. are supported by the National Institutes of Health NRSA fellowships F31-EY030409 and F31-EY031580, respectively. K.A.D. is supported by National Institutes of Health BUILD EXITO Program (TL4-GM118965) and Berkeley Molecular Biophysics Training Grant (T32-GM008295). C.C.Y. is supported by OHSU. J.C. and D.M.Z. are supported by the OHSU Center for Spatial Systems Biomedicine, by the National Science Foundation (MCB 1715823), and by the National Institutes of Health (R01-GM115805). S.L.R. is supported by the National Institutes of Health (R35-GM124779).

\section{Author contributions}

J.A.F., B.G.H., and K.A.D. contributed equally. K.A.D. and J.A.F. conducted the protein purification and reconstitution of CryoEM specimens. J.A.F. collected the CryoEM datasets, performed image analysis and atomic modeling. K.A.D., J.B.M., and C.C.Y. contributed to image analysis. B.G.H. conducted and analyzed the MD simulations. B.G.H., J.C., and D.M.Z contributed to the experimental design and analysis of MD simulations. All authors contributed to manuscript preparation. S.L.R. provided overall guidance to the design and execution of the work.

\section{Competing interests}

The authors declare no competing interests.

\section{Additional information}

Supplementary information is available for this paper at https://doi.org/10.1038/s41467020-18120-5.

Correspondence and requests for materials should be addressed to S.L.R.

Peer review information Nature Communications thanks the anonymous reveiwers for their contributions to the peer review of this work.

Reprints and permission information is available at http://www.nature.com/reprints

Publisher's note Springer Nature remains neutral with regard to jurisdictional claims in published maps and institutional affiliations.

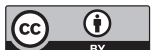

Open Access This article is licensed under a Creative Commons Attribution 4.0 International License, which permits use, sharing adaptation, distribution and reproduction in any medium or format, as long as you give appropriate credit to the original author(s) and the source, provide a link to the Creative Commons license, and indicate if changes were made. The images or other third party material in this article are included in the article's Creative Commons license, unless indicated otherwise in a credit line to the material. If material is not included in the article's Creative Commons license and your intended use is not permitted by statutory regulation or exceeds the permitted use, you will need to obtain permission directly from the copyright holder. To view a copy of this license, visit http://creativecommons.org/ licenses/by/4.0/

(c) The Author(s) 2020 